\title{
Concurrent OCT and OCT angiography of retinal neurovascular degeneration in the 5XFAD Alzheimer's disease mice
}

\author{
Tae-Hoon Kim $\odot,{ }^{\mathrm{a}}$ Taeyoon Son, ${ }^{\mathrm{a}}$ Dieter Klatt, ${ }^{\mathrm{a}}$ and Xincheng Yao $\odot^{\mathrm{a}, \mathrm{b}, *}$ \\ ${ }^{a}$ University of Illinois at Chicago, Department of Bioengineering, Chicago, Illinois, \\ United States \\ ${ }^{b}$ University of Illinois at Chicago, Department of Ophthalmology and Visual Sciences, \\ Chicago, Illinois, United States
}

\begin{abstract}
Significance: As one part of the central nervous system, the retina manifests neurovascular defects in Alzheimer's disease (AD). Quantitative imaging of retinal neurovascular abnormalities may promise a new method for early diagnosis and treatment assessment of AD. Previous imaging studies of transgenic AD mouse models have been limited to the central part of the retina. Given that the pathological hallmarks of $\mathrm{AD}$ frequently appear in different peripheral quadrants, a comprehensive regional investigation is needed for a better understanding of the retinal degeneration associated with AD-like pathology.
\end{abstract}

Aim: We aim to demonstrate concurrent optical coherence tomography (OCT) and OCT angiography (OCTA) of retinal neuronal and vascular abnormalities in the 5XFAD mouse model and to investigate region-specific retinal degeneration.

Approach: A custom-built OCT system was used for retinal imaging. Retinal thickness, vessel width, and vessel density were quantitatively measured. The artery and vein (AV) were classified for differential $\mathrm{AV}$ analysis, and trilaminar vascular plexuses were segmented for depth-resolved density measurement.

Results: It was observed that inner and outer retinal thicknesses were explicitly reduced in the dorsal and temporal quadrants, respectively, in 5XFAD mice. A significant arterial narrowing in 5XFAD mice was also observed. Moreover, overall capillary density consistently showed a decreasing trend in 5XFAD mice, but regional specificity was not identified.

Conclusions: Quadrant- and layer-specific neurovascular degeneration was observed in 5XFAD mice. Concurrent OCT and OCTA promise a noninvasive method for quantitative monitoring of $\mathrm{AD}$ progression and treatment assessment.

(C) The Authors. Published by SPIE under a Creative Commons Attribution 4.0 Unported License. Distribution or reproduction of this work in whole or in part requires full attribution of the original publication, including its DOI. [DOI: 10.1117/1.NPh.8.3.035002]

Keywords: Alzheimer's disease; retina; OCT; OCTA; neurovascular degeneration; 5XFAD.

Paper 21013RR received Mar. 24, 2021; accepted for publication Jun. 25, 2021; published online Jul. 10, 2021.

\section{Introduction}

Alzheimer's disease (AD) is a progressive neurodegenerative disease and the fifth leading cause of death in Americans over 65 years. ${ }^{1}$ The death rate resulting from AD soared $123 \%$ between 2000 and $2015,{ }^{1}$ and it is anticipated that one in every 85 people will be living with AD by $2050 .{ }^{2}$ There is no cure for $\mathrm{AD}$ to date; thus, early detection and therapeutic interventions are imperative to prolong cognitive function in $\mathrm{AD}$ patients. ${ }^{3,4} \mathrm{~A}$ definitive diagnosis of $\mathrm{AD}$ can be achieved by

*Address all correspondence to Xincheng Yao, xcy@uic.edu 
cerebrospinal fluid assays or positron emission tomography imaging. ${ }^{5,6}$ However, these methods are often invasive, costly, time-consuming, and limited in their availability.

There is ample evidence to support that the retina and brain share common pathological hallmarks of $\mathrm{AD},{ }^{7,8}$ highlighting the potential usefulness of retinal examination for $\mathrm{AD}$ diagnosis. Beta-amyloid $(\mathrm{A} \beta)$ deposits in the retina were found to be associated with brain $\mathrm{A} \beta$ burden, ${ }^{9,10}$ and morphological and functional impairments of the retina were also observed in $\mathrm{AD}$ patients. ${ }^{11,12}$ Given the clear optics of the eye, the retina offers easy accessibility to ocular imaging modalities, enabling noninvasive, cost-effective, and rapid screening to define at-risk AD populations. Accordingly, over the past decade, substantial progress has been made in retinal imaging techniques, such as retinal fluorescent imaging ${ }^{13}$ fluorescence lifetime imaging, ${ }^{14}$ hyperspectral imaging, ${ }^{15}$ spectrophotometric fundus imaging, ${ }^{16}$ optical coherence tomography (OCT) ${ }^{17}$ and OCT angiography (OCTA), ${ }^{18}$ to detect AD-associated abnormalities.

With depth-resolved capabilities, OCT and OCTA have been mostly used in clinical studies. OCT illustrated morphological abnormalities in the retina associated with $\mathrm{AD},{ }^{17}$ and OCTA demonstrated functional abnormalities in the retinal vasculature in AD patients. ${ }^{18}$ However, the clinical results often conflicted with each other, ${ }^{19,20}$ and age-related confounding factors cannot be easily excluded in measurement, ${ }^{21,22}$ limiting reliable interpretations for retinal changes associated with $\mathrm{AD}$. To better understand the causation of $\mathrm{AD}$ in the retina, studies using transgenic mouse models, recapitulating amyloid plaques, neurofibrillary tangles, or neurodegeneration, ${ }^{23}$ have been recently demonstrated. Georgevsky et al. ${ }^{24}$ used spectral-domain OCT in APP/PS1 mice and observed significant inner retinal thinning at 9 months and outer retinal thinning at 12 months. Harper et al. ${ }^{25}$ used multicontrast OCT in APP/PS1 mice and found that retinal vessel density and retinal thickness were comparable between the transgenic mice and control mice at 54 to 103 weeks old. Song et al. conducted an ex vivo study using OCT and angle-resolved lowcoherence interferometry in 3xTG-AD mice at 15 to 16 months of age. They found significant nerve fiber layer (NFL) thinning and scattering parameters changes on the NFL in 3xTG-AD mice. ${ }^{26}$ Lim et al. ${ }^{27}$ used spectral-domain OCT in 5XFAD mice and found significant NFL thinning at 6,12 , and 17 months of age.

The previous animal studies well demonstrated comparative results between transgenic and control mice; however, their quantitative analysis was mainly performed at the central retina, i.e., the optic nerve head $(\mathrm{ONH})$ region. Since pathological hallmarks frequently appear in different peripheral quadrants of the human retina in $\mathrm{AD},{ }^{13,20,21,28,29}$ comprehensive regional monitoring would provide better insights into AD-associated retinal degeneration. Moreover, comparative imaging of retinal neuronal and vascular systems may become a useful tool to study pathological mechanisms of AD-associated retinal degeneration in different mouse models. In this study, concurrent OCT/OCTA monitoring of retinal neuronal and vascular abnormalities in all retinal quadrants (up to $\sim 30$ - deg eccentricity from the ONH) in the 5XFAD mouse model of AD was first-time demonstrated. A clear trend toward a reduction in both retinal thickness and vascular parameters was found in different regions of the 5XFAD mouse retina.

\section{Materials and Methods}

\subsection{Animals}

Six-month-old female 5XFAD mice $(N=5)$ and B6SJLF1/J mice (WT; $N=6)$ were used in this study. 5XFAD mice overexpress mutated versions of the human amyloid precursor protein (APP) and human presenilin- 1 , resulting in $\mathrm{A} \beta$ plaques in the brain and retina ${ }^{30} \mathrm{It}$ is well documented that 6-month-old 5XFAD mice showed behavioral deficits,${ }^{31}$ neuronal death ${ }^{32}$ capillary stalling, ${ }^{33}$ and abnormal tissue viscoelasticity in brain subregions. ${ }^{34}$ All the mice were directly obtained from the Mutant Mouse Resource and Research Center (MMRRC Stock No. 34840JAX; Jackson Laboratory, Bar Harbor, Maine) after genotyping for the Pde $6 \mathrm{~b}^{\text {rd1 }}$ mutation. Thus, the 5XFAD mice homozygous for the recessive Pde $6 \mathrm{~b}^{\text {rd1 }}$ allele were not included in this study to ensure that visual impairments did not affect results. 


\subsection{Imaging System}

A custom-designed spectral-domain OCT system was used in this study. The system has been employed in multiple functional OCT/OCTA studies ${ }^{35-37}$ Briefly, a near-infrared superluminescent diode $(\lambda=810 \mathrm{~nm} ; \Delta \lambda=100 \mathrm{~nm}$; D-810-HP, Superlum, Carrigtwohill, County Cork, Ireland) was used as a light source. A line CCD camera with 2048 pixels (AViiVA EM4; e2v Technologies, Chelmsford, United Kingdom) was used for recording OCT spectra in the custom-built OCT spectrometer. The frame rate of the camera was set to $50 \mathrm{kHz}$. The axial and lateral resolutions were theoretically estimated as 2.9 and $11 \mu \mathrm{m}$, respectively. The measured axial resolution from the scattering profile of the external limiting membrane (ELM) was $3.4 \mu \mathrm{m}$. The ELM is the thin line of junctional complexes between Müller cells and photoreceptors $(<1 \mu \mathrm{m}$ thickness), which can be well described by a Gaussian to confirm the axial point spread function. ${ }^{38} 1-\mathrm{mW}$ power was illuminated on the mouse cornea.

\subsection{Experimental Procedures}

Anesthesia was intraperitoneally induced by a mixture of ketamine (100 mg/kg body weight) and xylazine $(5 \mathrm{mg} / \mathrm{kg}$ body weight), and a drop of $1 \%$ tropicamide ophthalmic solution (Akorn, Lake Forest, Illinois) was applied to the imaging eye. Next, a cover glass (12-54580; Microscope cover glass, Fisherbrand, Waltham, Massachusetts) with a drop of eye gel (Severe; GenTeal, Novartis, Basel, Switzerland) was placed on the imaging eye. After the mouse was fully anesthetized, the head was fixed by a bite bar and ear bar in the animal holder that provided five degrees of freedom (i.e., $x, y, z$, pitch, and roll). Volumetric raster scans were individually acquired from each retinal quadrant (dorsal, nasal, temporal, ventral quadrant, and the $\mathrm{ONH}$ ). The $\mathrm{ONH}$ region was first captured, which can serve as a central point of the retina, and the dorsal/ventral quadrants were captured, followed by the nasal/temporal quadrants. Four repeated B-scans at each slow-scan position were collected for OCTA construction; thus, each OCT volume consisted of $4 \times 600 \times 600$ A-scans. All animal experiments were approved by the local animal care and biosafety office and performed following the protocols approved by the Animal Care Committee (ACC) at the University of Illinois at Chicago (ACC Number: 19-044). This study followed the Association for Research in Vision and Ophthalmology Statement for the Use of Animals in Ophthalmic and Vision Research.

\subsection{OCTA Image Processing}

The OCTA images were constructed by implementing intensity-based speckle variance processing. ${ }^{39}$ Next, retinal flattening was performed by realigning each A-line. Three vascular layers were then manually segmented for vessel density analysis (Fig. 1), i.e., the superficial vascular plexus (SVP), intermediate capillary plexus (ICP), and deep capillary plexus (DCP). ${ }^{40}$ For each segmented layer, en face OCTA image was reconstructed by maximum intensity projection, and a Hessian-based vessel enhancement method proposed by Jerman was used to enhance vasculature in each en face image. ${ }^{41}$ After that, binarization for the SVP was done by adaptive thresholding, and binarization for the ICP and DCP was done by ridge-based vessel detection method. ${ }^{42}$ All binarized images were further processed by morphological opening operation to remove small particle noises. Image processing was performed on MATLAB R2016a (MathWorks, Natick, Massachusetts), in coordination with image processing package available in Fiji software. ${ }^{43}$

\subsection{Data and Statistical Analysis}

Retinal thickness, vascular width, and vascular density were measured for quantitative comparison. For thickness measurement, flattened OCT-B scans were prepared, and large vessels were manually removed to measure the retinal thickness solely [Fig. 1(k)]. Central 200 A-lines were averaged in the horizontal direction, returning one averaged intensity profile [Fig. 1(1)]. The regions in where the vessels were manually removed [Fig. 1(k)] were excluded from the averaging. Based on the average profile, the NFL, inner retinal layer, outer retinal layer, and total retinal thickness were manually measured [Fig. 1(m)]. For vascular width measurement, 

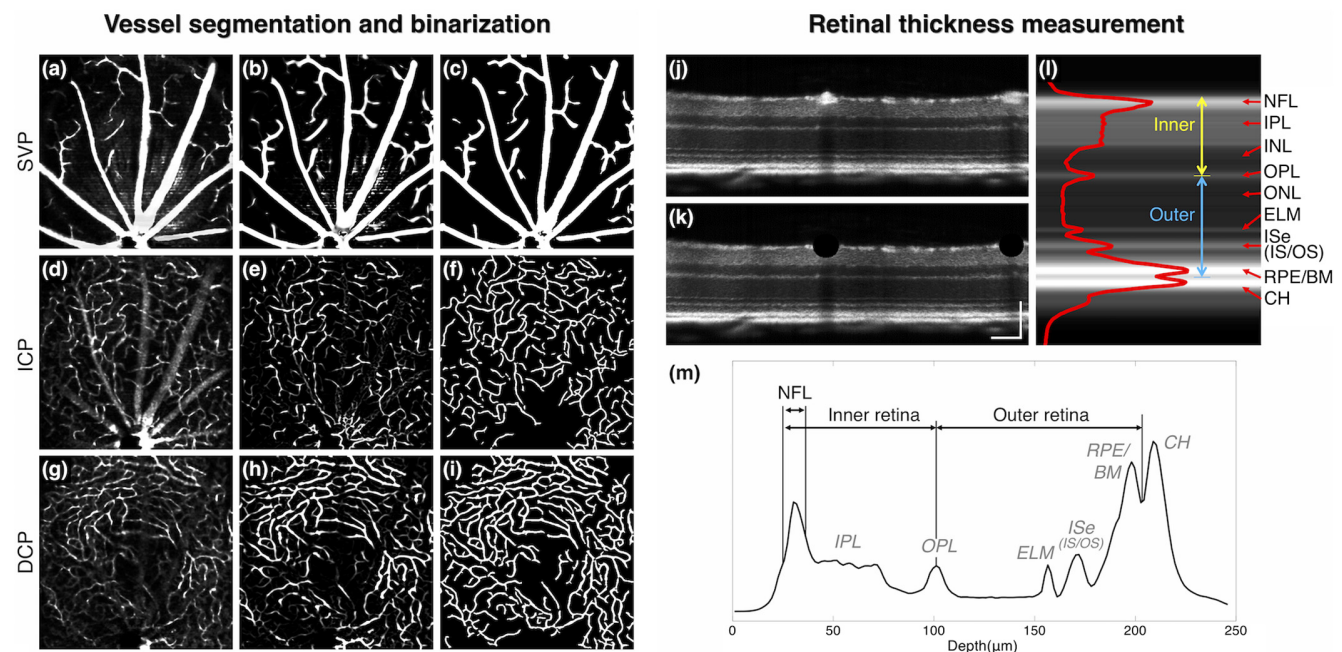

(m)

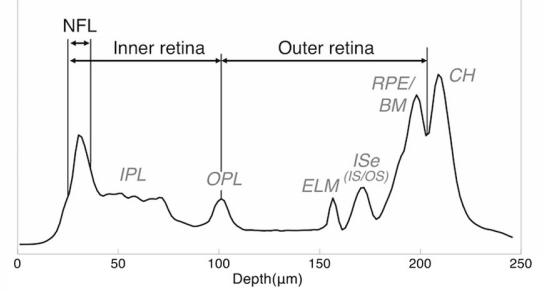

Artery-Vein classification
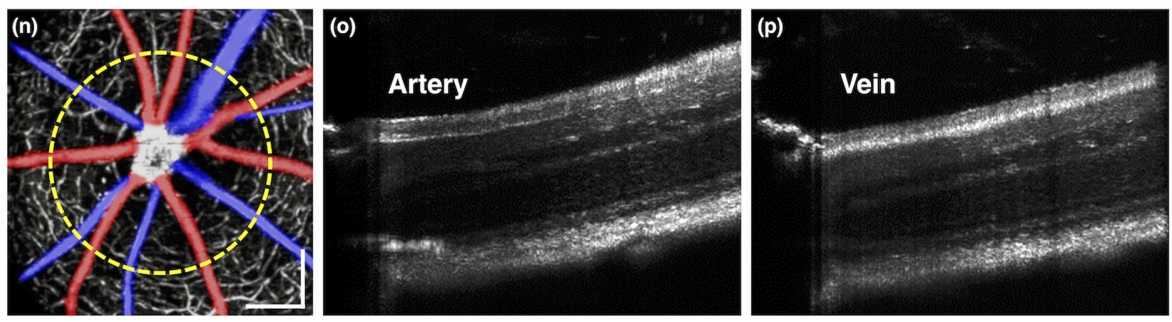

Fig. 1 OCTA binarization procedures. Representative en face OCTA of (a) SVP, (d) ICP, and (g) DCP in dorsal quadrant of the mouse retina. (b), (e), (h) Vessel enhancement was performed, followed by (c), (f), (i) binarization processing. (j) Flattened OCT B-scan. (k) Flattened OCT B-scan after vessel removal. Scale bars: $100 \mu \mathrm{m}$. (I) Representative A-line intensity profile. (m) Thickness measurement points. Midpoints of ascending and descending slopes of the NFL intensity profile, the OPL peak point, and the RPE/CH trough point were used in the measurement. (n) Pseudocolored en face OCTA image (red for arteries and blue for veins). Vessel width was measured at FWHM of the intensity profile of the yellow dashed circle. Scale bars: $200 \mu \mathrm{m}$. Representative pseudoradial OCT B-scans illustrate the different reflectance profiles between the (o) artery and ( $p$ ) vein. NFL, nerve fiber layer; IPL, inner plexiform layer; INL, inner nuclear layer; OPL, outer plexiform layer; ONL, outer nuclear layer; ELM, external limiting membrane; ISe, inner segment ellipsoid; IS, inner segment; OS, outer segment; RPE, retinal pigment epithelium; BM, Bruch's membrane; $\mathrm{CH}$, choroid.

the artery and vein (AV) around the $\mathrm{ONH}$ were first classified by referring vascular morphology on radially resliced OCT B-scans, ${ }^{44}$ and the vessel width of all first branches was measured based on the circular profile, $300 \mu \mathrm{m}$ away from the ONH [Figs. 1(n)-1(p)]. The full-width at halfmaximum (FWHM) was measured using the "findpeaks" function (MATLAB R2016a, MathWorks, Natick, Massachusetts) in determining the vessel diameter. The vessel density for each plexus was defined as the percentage of area occupied by vasculature in the binarized OCTA images. The two-sample $t$-test was performed for the statistical comparison between WT and 5XFAD, and a p-value $<0.05$ was considered statistically significant. In this study, each quadrant measurement was treated as an independent sample based on the current understanding that $\mathrm{AD}$-associated retinal degeneration appears in a specific retinal region, and this local effect does not affect measurements for other quadrant observations. ${ }^{20,21}$ Statistical analysis was performed on Origin 2020b (OriginLab, Northampton, Massachusetts).

\section{Results}

Figure 2 shows wide-field OCT and OCTA montages, consisting of four retinal quadrant images of a WT and 5XFAD mouse. Total imaging area covers $\sim 2.2 \mathrm{~mm} \times 2.2 \mathrm{~mm}$ of the central retina, 


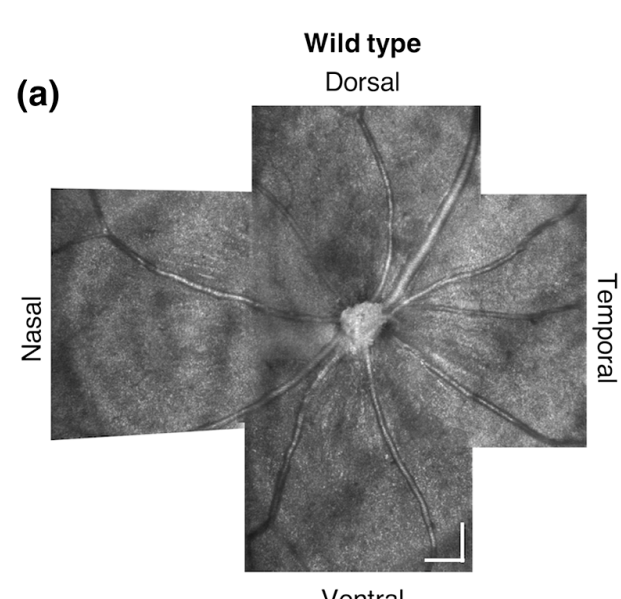

Ventral

(c)

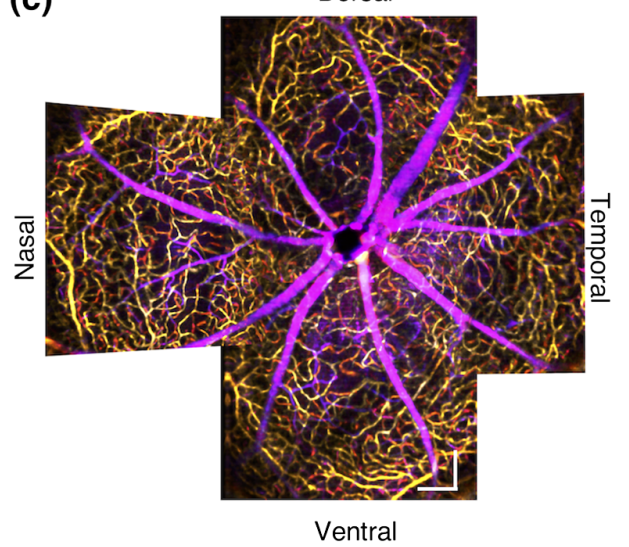

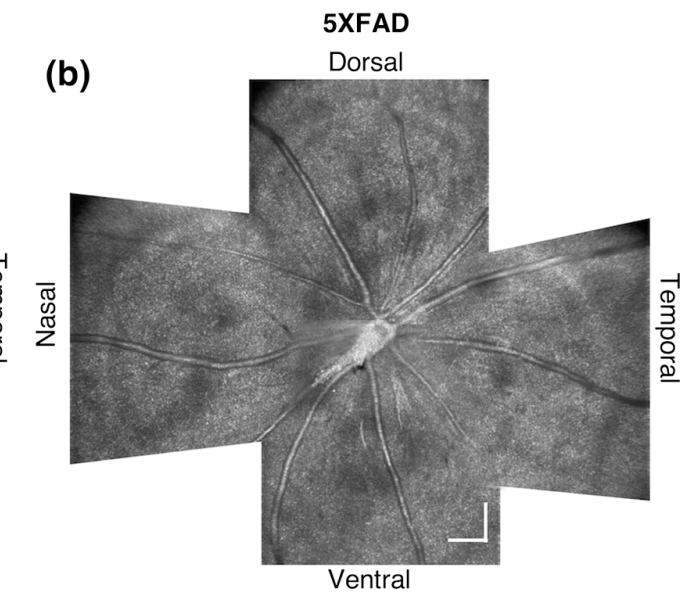

(d)

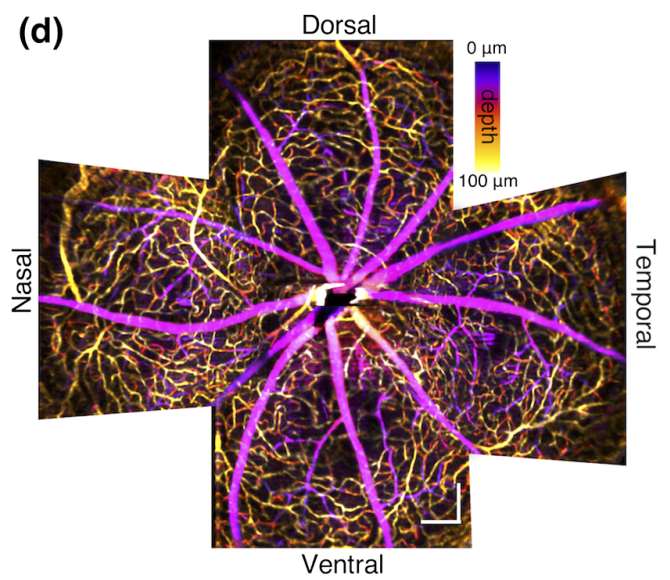

Fig. 2 Wide-field OCT and OCTA montages of (a), (c) the WT and (b), (d) the 5XFAD mouse retina. The color bar indicates the depth scale from the retinal surface to the outer plexiform layer. Scale bars: $200 \mu \mathrm{m}$.

which is $\sim 61$ - deg visual angle by the conversion factor of $36 \mu \mathrm{m}$ per degree of visual angle. The conversion factor was estimated based on a recent work demonstrating the adult mouse eye model incorporated with a contact lens and gel thickness information. ${ }^{45}$ This field of view can partially visualize network topologies of the mouse retina vasculature. Arteries and veins radially emanate from the $\mathrm{ONH}$, whereas the capillary plexus reveals a circular mesh structure centered at the ONH. ${ }^{46}$ Retinal abnormalities were not recognized between the WT and 5XFAD mouse through visual inspection, implying only subtle changes might occur in the 5XFAD mouse retina. Each quadrant OCT and OCTA volume was then individually processed and analyzed for the quantitative assessment.

We first analyzed neuronal change by retinal thickness measurement. The thickness of the NFL, inner retina, and outer retina were separately measured at the consistent position, $\sim 600 \mu \mathrm{m}$ away from the $\mathrm{ONH}$ in each quadrant. Overall, the NFL thickness (WT: $12.4 \pm 1.6 \mu \mathrm{m}$; 5XFAD: $11.2 \pm 2.0 \mu \mathrm{m} ; p=0.042$ ), inner retinal thickness (WT: $79.8 \pm 2.3 \mu \mathrm{m} ; 5 X F A D$ : $77.9 \pm 3.1 \mu \mathrm{m} ; p=0.033$ ), and outer retinal thickness (WT: $101.3 \pm 2.3 \mu \mathrm{m} ; 5 X F A D$ : $98.7 \pm 2.4 \mu \mathrm{m} ; p=0.001$ ) were significantly reduced in 5XFAD mice (Fig. 3). Accordingly, total retinal thickness was significantly lower in 5XFAD mice compared with WT mice (WT: $191.2 \pm 3.8 \mu \mathrm{m}$; 5XFAD: $188.0 \pm 4.1 \mu \mathrm{m} ; p=0.014$ ). Quadrant analysis also showed a consistent retinal thinning in different regions. There seems to be a trend that the dorsal area in the inner retina showed an advanced retinal thinning compared with the ventral side. The NFL in the dorsal quadrant was notably thinner in 5XFAD mice (WT: $13.4 \pm 2.0 \mu \mathrm{m}$; 5XFAD: $10.8 \pm 2.1 \mu \mathrm{m} ; p=0.063$ ) [Fig. 3(e)]. Another notable observation is the reduction of the outer retinal thickness. All quadrants showed a reduction in the outer retina, and the difference in the temporal quadrant was statistically significant (WT: $101.1 \pm 2.9 \mu \mathrm{m}$; 5XFAD: $97.4 \pm 1.3 \mu \mathrm{m}$; 
Kim et al.: Concurrent OCT and OCT angiography of retinal neurovascular...
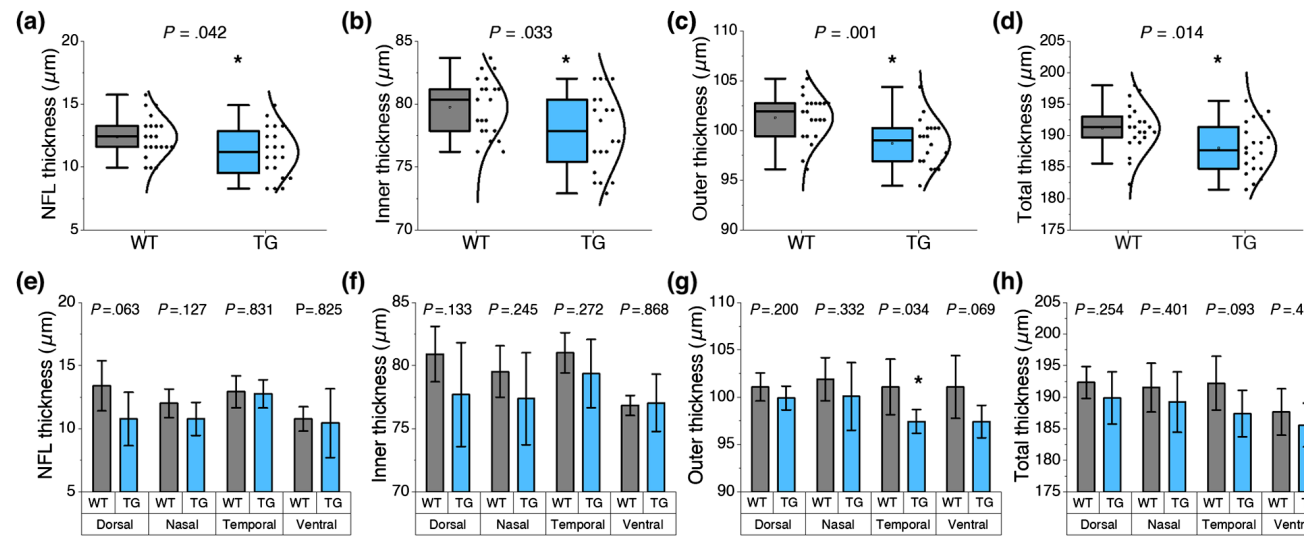

(g) (h)

(h) ${ }^{205}{ }^{2} P=.254 \quad P=.401 \quad P=.093 \quad P=.414$

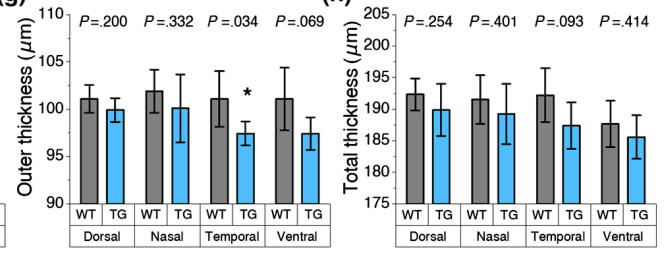

Fig. 3 Comparative retinal thickness measurement. (a) Overall NFL thickness and (e) quadrant specific measurement. (b) Overall inner retinal thickness and (f) quadrant-specific measurement. (c) Overall outer retinal thickness and (g) quadrant-specific measurement. (d) Overall total retinal thickness and (h) quadrant-specific measurement. Black dots in (a)-(d) indicate individual data points measured from each retinal quadrant. Data are expressed as mean \pm standard deviation per group. A two-sample $t$-test was performed for intergroup comparison. $P$-values are indicated in each plot, and statistical significance is indicated by an asterisk: ${ }^{*} P<0.05$. $N=6$ for WT (wild-type) and $N=5$ for TG (transgenic 5XFAD).

$p=0.034)[$ Fig. 3(g)]. Collectively, retinal thinning in all quadrants was clearly demonstrated in 6-month-old 5XFAD mice, and retinal thinning was attributed not only to the inner retina but also to the outer retina.

We next investigated vascular changes. To quantify the vascular narrowing/widening effect, we measured arterial and venular width following the circular profile around the $\mathrm{ONH}$ with radii of $300 \mu \mathrm{m}$. AV classification was achieved by the pseudoradial scanning method. ${ }^{44}$ Figures $1(\mathrm{o})$ and 1(p) show the radial OCT B-scans showing hyperreflective wall boundaries in retinal arteries, whereas these wall boundaries were absent in retinal veins. We found a significant arterial narrowing in 5XFAD mice (WT: $33.6 \pm 4.7 \mu \mathrm{m}$; 5XFAD: $28.3 \pm 4.8 \mu \mathrm{m} ; p<0.0001$ for average arterial width comparison, $p=0.007$ for average arterial width per mouse comparison, i.e., the average of the mean of each mouse dataset) [Fig. 4(a)], and the venular width was comparable between WT and 5XFAD mice (WT: $33.9 \pm 10.6 \mu \mathrm{m}$; 5XFAD: $31.1 \pm 10.1 \mu \mathrm{m}$; $p=0.309$ for average venular width comparison, $p=0.201$ for average venular width per mouse comparison, i.e., the average of the mean of each mouse dataset) [Fig. 4(b)]. The venular width exhibited more variations than the arterial width and did not show significant alterations between the two strains.
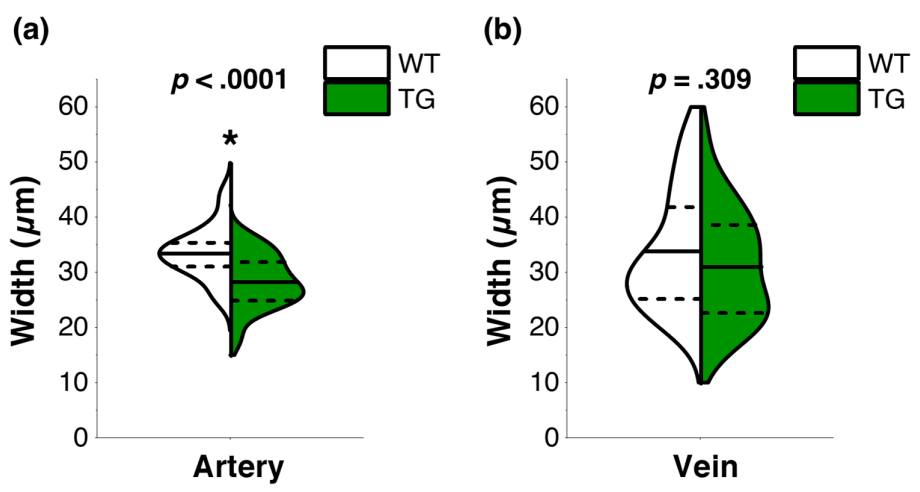

Fig. 4 Split-violin plots of (a) arterial width measurement and (e) venular width measurement. Data are expressed as mean \pm standard deviation per group. A two-sample $t$-test was performed for intergroup comparison. $P$-value for statistical significance is indicated by an asterisk. ${ }^{\star} P<0.05$. $N=6$ for WT (wild-type) and $N=5$ for TG (transgenic 5XFAD). 
(a)

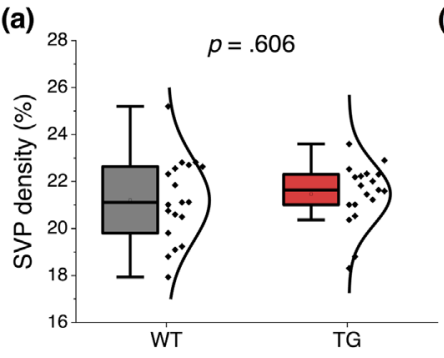

(d)

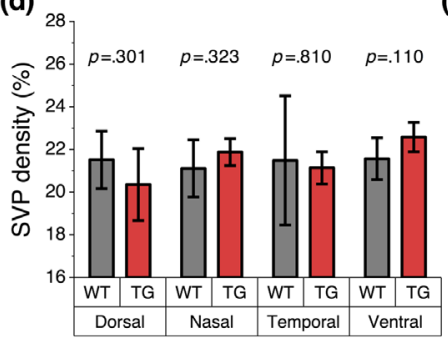

(b)

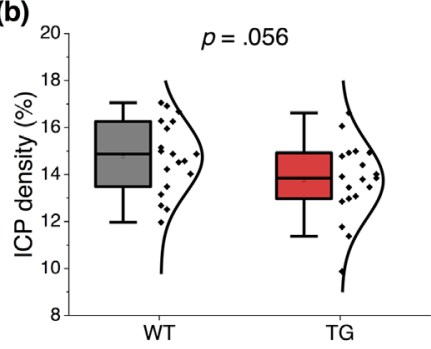

(e)

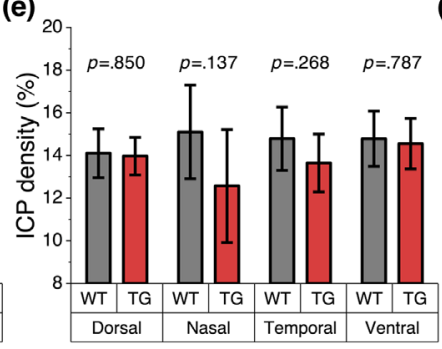

(c)

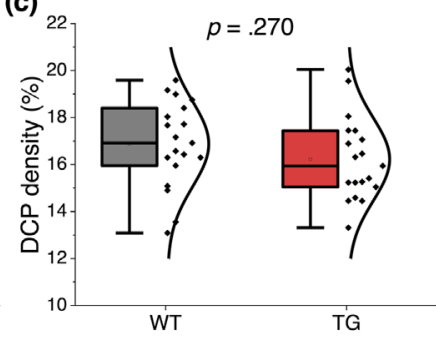

(f)

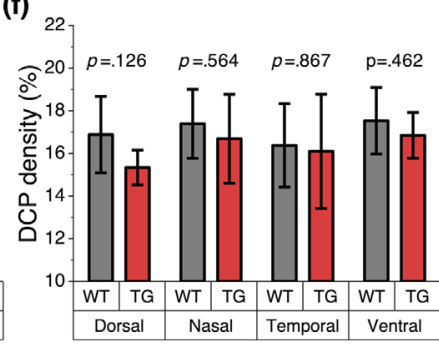

Fig. 5 Comparative vessel density measurement. (a) Overall SVP density and (d) quadrant-specific measurement. (b) Overall ICP density and (e) quadrant-specific measurement. (c) Overall DCP density and (f) quadrant-specific measurement. Black dots in (a)-(c) indicate individual data points measured from each retinal quadrant. Data are expressed as mean \pm standard deviation per group. A two-sample $t$-test was performed for intergroup comparison. $P$-values are indicated in each plot. $N=6$ for WT (wild-type) and $N=5$ for TG (transgenic 5XFAD).

Next vascular density was analyzed. The vasculature observed in OCTA reflects functional vessels. ${ }^{47}$ To measure the layer-specific change, trilaminar vascular plexuses were segmented (Fig. 1). Figure 5 shows that the vessel density in the SVP was comparable between WT and 5XFAD mice (WT: $21.2 \pm 1.8 \%$; 5XFAD: $21.4 \pm 1.3 \% ; p=0.606$ ), whereas the overall capillary density showed a decreasing trend in 5XFAD mice. Especially, the ICP density reduction in 5XFAD mice was close to being statistically significant (WT: $14.7 \pm 1.5 \%$; 5XFAD: $13.7 \pm 1.6 \% ; p=0.056$ ) [Fig. 5(b)]. We also found that capillary density consistently showed a decreasing trend in all retinal quadrants, although quadrant specificity tends to vary [Figs. 5(e) and 5(f)]. Collectively, the results demonstrate that the arterial narrowing effect was significant in 5XFAD mice, and functional capillary density in all quadrants was reduced in 5XFAD mice.

\section{Discussion}

In this study, region-specific neurovascular degeneration was found in 6-month-old 5XFAD mice. Significant inner retinal thinning was first noted in 5XFAD mice (Fig. 3), consistent with a recent finding of selective inner retinal deficits in 5XFAD mice at 6 months. ${ }^{27}$ The retinal thinning generally indicates neuronal death, ${ }^{48}$ and increasing evidence suggests that $\mathrm{A} \beta$ deposits in the inner retina may be associated with degeneration of retinal ganglion cells. ${ }^{49}$ Especially, the 5XFAD mouse model was found with the highest concentration of $\mathrm{A} \beta$ peptides in the brain and retina among $\mathrm{AD}$ mouse models. ${ }^{50}$ Although the exact role of $\mathrm{A} \beta$ in $\mathrm{AD}$ pathology remains an open question, it is believed that aggregation of extracellular $\mathrm{A} \beta$ can disrupt cellular communication and activities, which can damage synapses and ultimately induce neuronal cell death. ${ }^{51}$ In a recent study, notable $\mathrm{A} \beta$ accumulation was found around degenerating retinal ganglion cells in AD subjects. ${ }^{9}$ Also, we confirmed consistent outer retinal thinning in all quadrants [Fig. 3(g)]. The reason can be attributed to aggressive $\mathrm{A} \beta$ deposition not only in the inner retina but also in the outer retina in 5XFAD mice. It was reported that $\mathrm{A} \beta$ was largely accumulated in the 5XFAD retina under the retinal pigment epithelium (RPE), which might cause inflammation and contribute to photoreceptor cell death. ${ }^{52,53}$ Likewise, increased $\mathrm{A} \beta$ deposits around the rod photoreceptors were recently observed in $\mathrm{AD}$ subjects along with outer retinal thinning. ${ }^{13,54}$ The presence of $\mathrm{A} \beta$ may cause defects in photoreceptor cell function and potentially contribute 
to visual impairments in $\mathrm{AD}$ subjects as reflected by the reduction of contrast sensitivity, visual acuity, color vision, and visual field. ${ }^{55}$

Six-month-old 5XFAD mice also manifested vascular abnormalities in the retina. Arteries showed a significant narrowing in 5XFAD mice [Fig. 4(a)], which might be a consequence of the capillary flow deficits. Although capillary density reduction did not reach statistical significance, the ICP and DCP consistently exhibited a decreasing trend in all quadrants of the 5XFAD mouse retina [Figs. 5(b) and 5(c)]. Hernández et al. ${ }^{33}$ found that capillary stalling was elevated in 6-month-old 5XFAD mice and described that a small portion of capillary occlusion could dramatically decrease blood flow in up-and-down-stream vessels, resulting in the blood flow changes on cerebral blood flow. Nortley et al. also suggested that capillaries could be the most critical locus where $\mathrm{A} \beta$ can decrease cerebral blood flow, ${ }^{56}$ and reduced blood flow was found to increase in $\mathrm{A} \beta$ deposition, suggesting that blood flow deficits can further worsen $\mathrm{A} \beta$ pathology. ${ }^{57}$ However, there is an opposite pathophysiological basis that angiogenesis can occur in response to impaired cerebral perfusion and vascular injury. ${ }^{58}$ Such inflammation due to $\mathrm{A} \beta$ accumulation can induce the retina to become hypoxic and trigger angiogenesis, ${ }^{19}$ which can cause increased vessel density observed in OCTA. ${ }^{59}$ A recent clinical study reported capillary density increase in AD subjects ${ }^{60}$ while others reported the opposite results. ${ }^{61,62}$ We speculate that capillary density may increase at the initial stage of the $\mathrm{A} \beta$ accumulation. However, at the later stage, when neuronal cell loss occurs, capillary density may naturally decrease due to a reduction of metabolic demand and damaged endothelial cells. A further longitudinal study is required to verify this hypothesis.

Individual quadrant OCT/OCTA analysis suggested that neurovascular degeneration may occur in all retinal regions, but there seems to be a trend of regional specificity in terms of degeneration progress. Retinal thinning of the NFL and outer retina was distinguished in the dorsal and temporal quadrant, respectively, in 5XFAD mice (Figs. 3 and 5). Song et al. ${ }^{26}$ also found different light scattering parameters only in the dorsal quadrant in $\mathrm{AD}$ mouse retinas. In fact, a region-specific degeneration is not only limited to the animal models but also appeared in human subjects with $\mathrm{AD} .{ }^{21}$ Previous studies demonstrated that retinal changes were predominantly focused on the superior and inferior regions of the NFL in AD subjects. ${ }^{28,29}$ Querques et al. ${ }^{12}$ reported that ganglion cell layer thickness was reduced in AD subjects only in the central and temporal retinal region. Koronyo et al. ${ }^{13}$ found that $\mathrm{A} \beta$ plaques mainly appeared in the periphery of the superior quadrant, whereas some regional changes were discovered in each retinal layer differentially. ${ }^{54}$ Such discrepancy requires further verification of region-specific changes in the retina associated with AD pathology.

This study has three limitations to be addressed. First, a relatively small sample size was used in this cross-sectional study, which naturally lowered the statistical power to draw a definitive conclusion. Prospective longitudinal monitoring with an increasing sample size would further elucidate retinal abnormalities associated with AD pathology in transgenic mouse models. Second, there was no conclusive biomarker confirmation. Postmortem histopathology would be helpful to confirm the presence of AD biomarkers, such as $\mathrm{A} \beta 42$, total tau (T-tau), and phosphorylated tau (P-tau), in different retinal regions to correlate the level of biomarkers with OCT/OCTA features. Third, it is important to be aware of anesthesia-related cardiovascular effects, including changes in blood pressures, cardiac output, and varying heart rhythms. The ketamine-xylazine mixture is known to have potent cardiodepressive effects that can potentially lower local vessel diameter and blood flow over time. ${ }^{63,64}$ Since the effect is timedependent, in this study, the ONH region was always first captured in each mouse, and vessel width measurement was performed around the ONH [Fig. 1(n)]. Monitoring vital signs during image acquisition would allow for better understanding of anesthesia-related cardiovascular effects on the mouse retina. Despite the limitations of this study, in vivo full quadrant imaging may provide a foundation for future detailed exploration of the relationship between retinal neurovascular defects and $\mathrm{AD}$-associated retinal degeneration.

\section{Conclusions}

This study demonstrates in vivo OCT/OCTA monitoring of all retinal quadrants, which allowed examining region-specific neurovascular degeneration in the 5XFAD mouse retina. 
Six-month-old 5XFAD mice revealed that inner and outer retinal degeneration were explicitly advanced in the dorsal and temporal quadrant, respectively. The arterial narrowing was observed in 5XFAD compared with wild-type mice. Overall decrease of measured capillary density was also observed in 5XFAD mice, suggesting a tight correlation between retinal neurons and vasculature. The proposed imaging strategy promises a noninvasive method for longitudinal monitoring of $\mathrm{AD}$ progression and treatment assessment in transgenic mouse models of $\mathrm{AD}$.

\section{Disclosures}

The authors declared no potential conflicts of interest with respect to the research, authorship, and/or publication of this article.

\section{Acknowledgments}

This research was supported in part by the National Institutes of Health (NIH) Grant Nos. R01EY023522, R01EY030101, R01EY030842, R01EY029673, and P30EY001792; by the Chicago Biomedical Consortium with support from the Searle Funds at the Chicago Community Trust; by the Richard and Loan Hill endowment; and by an unrestricted grant from Research to Prevent Blindness.

\section{Code, Data, and Materials Availability}

The data that support the findings of this study are available from the corresponding author upon reasonable request.

\section{References}

1. Alzheimer's Association, "2018 Alzheimer's disease facts and figures," Alzheimer's Dementia 14(3), 367-429 (2018).

2. C. L. Masters et al., "Alzheimer's disease," Nat. Rev. Disease Primers 1(1), 15056 (2015).

3. P. Aisen et al., "EU/US/CTAD task force: lessons learned from recent and current Alzheimer's prevention trials," J. Prev. Alzheimers Dis. 4(2), 116-124 (2017).

4. S. G. Gauthier, "Alzheimer's disease: the benefits of early treatment," Eur. J. Neurol. 12(s3), 11-16 (2005).

5. A. S. Fleisher et al., "Associations between biomarkers and age in the presenilin 1 E280A autosomal dominant Alzheimer disease kindred: a cross-sectional study," JAMA Neurol. 72(3), 316-324 (2015).

6. J. Weller and A. Budson, "Current understanding of Alzheimer's disease diagnosis and treatment," F1000Res 7, 1161 (2018).

7. C. Y.-I. Cheung et al., "Imaging retina to study dementia and stroke," Prog. Retinal Eye Res. 57, 89-107 (2017).

8. N. Mirzaei et al., "Alzheimer's retinopathy: seeing disease in the eyes," Front. Neurosci. 14, 921 (2020).

9. C. La Morgia et al., "Melanopsin retinal ganglion cell loss in Alzheimer disease," Ann. Neurol. 79(1), 90-109 (2016).

10. M. Koronyo-Hamaoui et al., "Identification of amyloid plaques in retinas from Alzheimer's patients and noninvasive in vivo optical imaging of retinal plaques in a mouse model," Neuroimage 54(Suppl.), S204-S217 (2011).

11. M. Chiasseu et al., "Tau accumulation in the retina promotes early neuronal dysfunction and precedes brain pathology in a mouse model of Alzheimer's disease," Mol. Neurodegener. 12(1), 58-58 (2017).

12. G. Querques et al., "Functional and morphological changes of the retinal vessels in Alzheimer's disease and mild cognitive impairment," Sci. Rep. 9(1), 63-63 (2019).

13. Y. Koronyo et al., "Retinal amyloid pathology and proof-of-concept imaging trial in Alzheimer's disease," JCI Insight 2(16), e93621 (2017). 
14. S. Jentsch et al., "Retinal fluorescence lifetime imaging ophthalmoscopy measures depend on the severity of Alzheimer's disease," Acta Ophthalmol. 93(4), e241-e247 (2015).

15. X. Hadoux et al., "Non-invasive in vivo hyperspectral imaging of the retina for potential biomarker use in Alzheimer's disease," Nat. Commun. 10(1), 4227 (2019).

16. A. B. Einarsdottir et al., "Retinal oximetry imaging in Alzheimer's disease," J. Alzheimer's Disease 49, 79-83 (2016).

17. V. T. T. Chan et al., "Spectral-domain OCT measurements in Alzheimer's disease: a systematic review and meta-analysis," Ophthalmology 126(4), 497-510 (2019).

18. L. Lahme et al., "Evaluation of ocular perfusion in Alzheimer's disease using optical coherence tomography angiography," J. Alzheimer's Disease 66, 1745-1752 (2018).

19. J. Ngolab, P. Honma, and R. A. Rissman, "Reflections on the utility of the retina as a biomarker for Alzheimer's disease: a literature review," Neurol. Ther. 8(Suppl.), 57-72 (2019).

20. V. B. Gupta et al., "Retinal changes in Alzheimer's disease-integrated prospects of imaging, functional and molecular advances," Prog. Retinal Eye Res. 82, 100899 (2020).

21. N. J. Hart et al., "Ocular indicators of Alzheimer's: exploring disease in the retina," Acta Neuropathol. 132(6), 767-787 (2016).

22. A. I. Ramirez et al., "The role of microglia in retinal neurodegeneration: Alzheimer's disease, Parkinson, and Glaucoma," Front Aging Neurosci. 9, 214-214 (2017).

23. J. L. Jankowsky and H. Zheng, "Practical considerations for choosing a mouse model of Alzheimer's disease," Mol. Neurodegener. 12(1), 89 (2017).

24. D. Georgevsky et al., "A longitudinal assessment of retinal function and structure in the APP/PS1 transgenic mouse model of Alzheimer's disease," Translational Neurodegener. 8(1), 30 (2019).

25. D. J. Harper et al., "Retinal analysis of a mouse model of Alzheimer's disease with multicontrast optical coherence tomography," Neurophotonics 7(1), 015006 (2020).

26. G. Song et al., "Multimodal coherent imaging of retinal biomarkers of Alzheimer's disease in a mouse model," Sci. Rep. 10(1), 7912 (2020).

27. J. K. H. Lim et al., "Retinal functional and structural changes in the 5xFAD mouse model of Alzheimer's disease," Front. Neurosci. 14, 862 (2020).

28. Y. Lu et al., "Retinal nerve fiber layer structure abnormalities in early Alzheimer's disease: evidence in optical coherence tomography," Neurosci. Lett. 480(1), 69-72 (2010).

29. A. López-de-Eguileta et al., "Ganglion cell layer thinning in prodromal Alzheimer's disease defined by amyloid PET," Alzheimers Dement (N Y) 5, 570-578 (2019).

30. H. Oakley et al., "Intraneuronal $\beta$-Amyloid aggregates, neurodegeneration, and neuron loss in transgenic mice with five familial Alzheimer's disease mutations: potential factors in amyloid plaque formation," J. Neurosci. 26(40), 10129 (2006).

31. S. Jawhar et al., "Motor deficits, neuron loss, and reduced anxiety coinciding with axonal degeneration and intraneuronal $\mathrm{A} \beta$ aggregation in the 5XFAD mouse model of Alzheimer's disease," Neurobiol. Aging 33(1), 196.e29-196.e40 (2012).

32. W. A. Eimer and R. Vassar, "Neuron loss in the 5XFAD mouse model of Alzheimer's disease correlates with intraneuronal A $\beta 42$ accumulation and Caspase-3 activation," Mol. Neurodegener. 8, 2 (2013).

33. J. C. Cruz Hernández et al., "Neutrophil adhesion in brain capillaries reduces cortical blood flow and impairs memory function in Alzheimer's disease mouse models," Nat. Neurosci. 22(3), 413-420 (2019).

34. S. Majumdar and D. Klatt, "Longitudinal study of sub-regional cerebral viscoelastic properties of 5XFAD Alzheimer's disease mice using multifrequency MR elastography," Magn. Reson Med. 86(1), 405-414 (2021).

35. T. Son et al., "Functional optical coherence tomography of neurovascular coupling interactions in the retina," J. Biophotonics 11(12), e201800089 (2018).

36. T.-H. Kim et al., "Longitudinal OCT and OCTA monitoring reveals accelerated regression of hyaloid vessels in retinal degeneration 10 (rd10) mice," Sci. Rep. 9(1), 16685 (2019).

37. G. Ma et al., "In vivo optoretinography of phototransduction activation and energy metabolism in retinal photoreceptors," J. Biophotonics 14(5), e202000462 (2021). 
38. R. F. Spaide and C. A. Curcio, "Anatomical correlates to the bands seen in the outer retina by optical coherence tomography: literature review and model," Retina 31(8), 1609-1619 (2011).

39. T. Son et al., "Optical coherence tomography angiography of stimulus evoked hemodynamic responses in individual retinal layers," Biomed. Opt. Express 7(8), 3151-3162 (2016).

40. T.-H. Kim et al., "Comparative optical coherence tomography angiography of wild-type and rd10 mouse retinas," Translational Vision Sci. Technol. 7(6), 42 (2018).

41. T. Jerman et al., "Enhancement of vascular structures in 3D and 2D angiographic images," IEEE Trans. Med. Imaging 35(9), 2107-2118 (2016).

42. C. Steger, "An unbiased detector of curvilinear structures," IEEE Trans. Pattern Anal. Mach. Intell. 20(2), 113-125 (1998).

43. J. Schindelin et al., "Fiji: an open-source platform for biological-image analysis," Nat. Methods 9, 676-682 (2012).

44. T.-H. Kim et al., "Vascular morphology and blood flow signatures for differential artery-vein analysis in optical coherence tomography of the retina," Biomed. Opt. Express 12(1), 367379 (2021).

45. P. Zhang et al., "Effect of a contact lens on mouse retinal in vivo imaging: effective focal length changes and monochromatic aberrations," Exp. Eye Res. 172, 86-93 (2018).

46. P. Ganesan, S. He, and H. Xu, "Development of an image-based model for capillary vasculature of retina," Comput. Methods Prog. Biomed. 102(1), 35-46 (2011).

47. T.-H. Kim, T. Son, and X. Yao, "Functional OCT angiography reveals early physiological dysfunction of hyaloid vasculature in developing mouse eye," Exp. Biol. Med. 244(10), 819-823 (2019).

48. C. La Morgia et al., "Patterns of retinal ganglion cell damage in neurodegenerative disorders: parvocellular vs magnocellular degeneration in optical coherence tomography studies," Front. Neurol. 8, 710 (2017).

49. C. Criscuolo et al., "The retina as a window to early dysfunctions of Alzheimer's disease following studies with a 5xFAD mouse model," Neurobiol. Aging 67, 181-188 (2018).

50. P. N. Alexandrov et al., "Retinal amyloid peptides and complement factor $\mathrm{H}$ in transgenic models of Alzheimer's disease," Neuroreport 22(12), 623-627 (2011).

51. Q. Li, Y. Liu, and M. Sun, “Autophagy and Alzheimer's disease," Cell. Mol. Neurobiol. 37(3), 377-388 (2017).

52. S. W. Park et al., "Dry age-related macular degeneration like pathology in aged 5XFAD mice: ultrastructure and microarray analysis," Oncotarget 8(25), 40006-40018 (2017).

53. K. V. Do et al., "Elovanoids counteract oligomeric $\beta$-amyloid-induced gene expression and protect photoreceptors," Proc. Natl. Acad. Sci. U. S. A. 116(48), 24317 (2019).

54. L. Jáñez-Escalada et al., "Spatial analysis of thickness changes in ten retinal layers of Alzheimer's disease patients based on optical coherence tomography," Sci. Rep. 9(1), 13000 (2019).

55. L. Rodriguez et al., "Human tau expression does not induce mouse retina neurodegeneration, suggesting differential toxicity of tau in brain vs. retinal neurons," Front. Mol. Neurosci. 11, 293 (2018).

56. R. Nortley et al., "Amyloid $\beta$ oligomers constrict human capillaries in Alzheimer's disease via signaling to pericytes," Science 365(6450), eaav9518 (2019).

57. L. Wang et al., "Chronic cerebral hypoperfusion induces memory deficits and facilitates A $\beta$ generation in C57BL/6J mice," Exp. Neurol. 283, 353-364 (2016).

58. A. H. Vagnucci and W. W. Li, “Alzheimer's disease and angiogenesis," Lancet 361(9357), 605-608 (2003).

59. D. C. Sousa et al., "Hypoxia challenge test and retinal circulation changes: a study using ocular coherence tomography angiography," Acta Ophthalmol. 96(3), e315-e319 (2018).

60. J. A. van de Kreeke et al., "Optical coherence tomography angiography in preclinical Alzheimer's disease,” Br. J. Ophthalmol. 104(2), 157 (2020).

61. S. P. Yoon et al., "Retinal microvascular and neurodegenerative changes in Alzheimer's disease and mild cognitive impairment compared with control participants," Ophthalmol. Retina 3(6), 489-499 (2019). 
62. H. Jiang et al., "Altered macular microvasculature in mild cognitive impairment and Alzheimer disease," J. Neuroophthalmol. 38(3), 292-298 (2018).

63. B. J. A. Janssen et al., "Effects of anesthetics on systemic hemodynamics in mice," Am. J. Physiol. Heart Circul. Physiol. 287(4), H1618-H1624 (2004).

64. E. M. Moult et al., "Evaluating anesthetic protocols for functional blood flow imaging in the rat eye," J. Biomed. Opt. 22(1), 016005 (2017).

Tae-Hoon Kim is a PhD candidate at the University of Illinois at Chicago (UIC). He received his BS and MS degrees in biomedical engineering from the Catholic University of Daegu, South Korea. After graduation, he worked at Texas Tech University for 1 year as a research scientist. He then joined the Yao Lab at UIC in August 2017 for his doctoral study. His current research focuses on in vivo functional optical imaging of the retina.

Taeyoon Son is a research assistant professor of bioengineering at the UIC. He received his $\mathrm{PhD}$ from Yonsei University in South Korea. His research focuses on optical imaging technology, including OCT, OCTA, and ultrawidefield fundus, super-resolution scanning laser ophthalmoscopy, and its application to ophthalmology.

Dieter Klatt is an associate professor of bioengineering at the UIC. He received his MS degree in geophysics from Ruhr-University Bochum, Germany, and his $\mathrm{PhD}$ in physics from Humboldt University Berlin, Germany. He has been an elected member of the governing committee of the MR Elastography Group within the International Society for Magnetic Resonance in Medicine (ISMRM) since 2017. His research involves the development of rapid motionencoding MRI acquisition approaches with a special focus on MR elastography and the examination of the diagnostic potential of motion-encoding MRI for a variety of diseases including neurodegeneration.

Xincheng Yao is a Richard \& Loan Hill professor of bioengineering and ophthalmology and visual sciences at the UIC. He received his PhD in optics from the Institute of Physics, Chinese Academy of Sciences, in 2001. This was followed by his postdoctoral research in the Biophysical Group at Los Alamos National Laboratory (LANL) from 2001 to 2004. He held a LANL technical staff appointment from 2004 to 2006 and served at CFD Research Corporation as a senior research scientist from 2006 to 2007 . He was a faculty member of the University of Alabama at Birmingham from 2007 to 2014 and joined the UIC faculty in 2014. His research interests include biomedical optics instrumentation, ultrawidefield fundus photography, functional OCT, OCT angiography, super-resolution ophthalmoscopy, and machine learning image classification. 